\title{
Association between Living Arrangements and Influenza Vaccination Rates among Elderly South Korean People: The Fifth Korea National Health and Nutrition Examination Survey (KNHANES V-2)
}

Hye Min Cho, Joo-Yeon Kim*, Seo Eun Hwang, Jae Chul Kim, Moo-Young Kim, Soo Hyoung Lee

Department of Family Medicine, Seoul Medical Center, Seoul, Korea

Background: This study aimed to investigate the association between living arrangements and influenza vaccination among elderly South Korean subjects.

Methods: We used data from the fifth Korean National Health and Nutrition Examination Survey. Participants older than 65 years were included and categorized into 4 groups according to the type of living arrangement as follows: (1) living alone group; (2) living with a spouse group; (3) living with offspring (without spouse) group; and (4) living with other family members group. A total of 1,435 participants were included in this cross-sectional analysis.

Results: A lower vaccination rate was observed in the living with offspring (without spouse) group, whereas the living with a spouse group had higher rates of both seasonal and H1N1 influenza vaccination. After adjusting for age, sex, region, education level, income level, and number of comorbidities, the living with offspring (without spouse) group had a higher $\mathrm{H} 1 \mathrm{~N} 1$ vaccination non-receipt rate than the living alone group (odds ratio, 2.03; 95\% confidence interval, 1.08-3.82).

Conclusion: Influenza vaccination rates differed according to the type of living arrangement. Particularly, those living with offspring (without spouse) had the lowest H1N1 influenza vaccination rate compared to those with other living arrangements, and this difference was significant. Interventions to improve influenza vaccination coverage should target not only elderly persons who live alone, but also those living with offspring.

Keywords: Influenza Vaccines; Vaccination; Residence Characteristics; Aged 


\section{INTRODUCTION}

Influenza is a worldwide public health problem. ${ }^{1)}$ This disease often causes severe complications such as pneumonia, the sixth-leading cause of death in Korea during the year 2013. ${ }^{2)}$ According to the World Health Organization, global influenza epidemics annually result in 3 to 5 million cases of severe illness and 250,000 to 500,000 deaths. The majority of deaths associated with influenza in industrialized countries occur among citizens aged 65 years or older. ${ }^{1)}$

Vaccination is the most effective way to prevent infection and severe outcomes caused by influenza viruses. ${ }^{3)}$ In particular, vaccination reduces morbidity and mortality among high-risk groups such as the elderly. ${ }^{4-6)}$ The factors associated with receiving vaccination include age, sex, educational level, household income, place of residence, contact with the health care system, hospitalization, and comorbidities. ${ }^{7-12)}$ Some studies observed a relationship between increasing age and an increasing vaccination rate, although the limited functional statuses of individuals in some older groups negatively affected the vaccination rate. A lower educational level and lower income were associated with lower vaccination coverage. Identification of the factors associated with vaccination in the elderly is required when determining which segments of the elderly population should be targeted to increase the overall coverage of influenza vaccination programs. ${ }^{9-14)}$ Living arrangements have also been associated with health status and health behaviors. A previous study found an association between the type of living arrangement and the use of preventive care such as influenza vaccination and hypertension screening. ${ }^{15)}$ In addition, some studies have suggested that married people have better health than do unmarried people. ${ }^{16,17)}$ However, an association between living arrangements and influenza vaccination has not been established for the Korean population. In this context, we investigated the association between living arrangements and influenza vaccination among a sample of elderly Korean individuals.

\section{METHODS}

\section{Study Population}

Data for this cross-sectional study were obtained from the second year (2011) of the fifth Korean National Health and Nutrition Examination Survey (KNHANES V-2), which was conducted by the Korean Centers for Disease Control and Prevention to assess the health and nutritional status of the South Korean population. KNHANES V-2 comprised a health examination survey; a comprehensive self-reported questionnaire of anthropometric and demographic characteristics, socioeconomic status, and comorbidities; and a nutrition survey. A geographic region based-multistage probability sampling, stratified according to sex and age, was used to select household units. A total of 8,518 individuals from these sampling frames were included in the 2011 survey. Among them, 1,598 individuals aged over 65 years were identified as possible participants in our study. We excluded those with missing data regarding living arrangements and influenza vaccine receipt. We also excluded participants for whom information on confounding variables (region, household income, education level, and chronic diseases) was missing. A total of 1,435 participants were ultimately eligible for this study. All study participants provided written informed consents. The study was approved by the institutional review board (IRB) of Korea Centers for Disease Control and Prevention (IRB: 2011-02CON-06-C).

\section{Variables}

Sixteen regions (Seoul, 6 metropolitan cities, and 9 provinces) were defined for KNHANES. In this study, the regions were recategorized into 3 groups (Seoul, metropolitan cities, and provinces). Household income levels were divided into quartiles, which were calculated based on equalized income (total household income divided by the square root of the number of people in a household). Education level was classified into 4 categories: completion of elementary school, middle school, high school, and post-secondary school. In this study, chronic diseases included hypertension, hyperlipidemia, stroke, angina, myocardial infarction, pulmonary tuberculosis, asthma, diabetes, and cancer. Seasonal influenza and H1N1 influenza vaccine receipt were self-reported separately as a yes response to a question on whether the participant had received the vaccination during the past year. Types of living arrangements were grouped into 4 categories according to the response to "what is your type of household?" These groups were living alone (answered "single person household"), living with a spouse (answered "living with a spouse only or a spouse and other family members, including offspring, parents, or others"), living with offspring (without a spouse; answered "living as a single parent without a spouse), and living with other family members (all others).

\section{Statistical Analyses}

Statistical analyses were conducted using SPSS statistical software ver. 12.0 (SPSS Inc., Chicago, IL, USA). One-way analysis of variance was used to compare the means of continuous variables among living arrangement groups. Categorical variables were assessed using the chi-square test. Logistic regression analyses were used to examine the association between living arrangements and influenza vaccination. All tests were two-sided, and a P-value $<0.05$ was considered statistically significant.

\section{RESULTS}

The participants' ages ranged from 65 to 97 years, with a mean age of 72.8 years, and $57.6 \%(n=827)$ of the participants were 
Table 1. Sociodemographic characteristics according to the type of living arrangement

\begin{tabular}{|c|c|c|c|c|c|}
\hline Variable & $\begin{array}{l}\text { Living alone } \\
\qquad(n=277)\end{array}$ & $\begin{array}{l}\text { Living with a spouse } \\
\qquad(n=925)\end{array}$ & $\begin{array}{l}\text { Living with offspring } \\
\qquad(\mathrm{n}=48)\end{array}$ & $\begin{array}{l}\text { Living with other family members } \\
\qquad(\mathrm{n}=185)\end{array}$ & P-value* \\
\hline Female & $230(83.0)$ & $429(46.4)$ & 45 (93.8) & $123(66.5)$ & $<0.001$ \\
\hline Age (y) & $74.55 \pm 5.65$ & $72.02 \pm 5.10$ & $72.98 \pm 5.64$ & $74.01 \pm 5.95$ & $<0.001$ \\
\hline Education $^{\dagger}$ & & & & & $<0.001$ \\
\hline Elementary school & 236 (85.2) & $565(61.1)$ & 38 (79.2) & $124(67.0)$ & \\
\hline Middle school & $22(7.9)$ & $120(13.0)$ & $3(6.2)$ & $21(11.4)$ & \\
\hline High school & $13(4.7)$ & $172(18.6)$ & $5(10.4)$ & $26(14.1)$ & \\
\hline Post-secondary school & $6(2.2)$ & $68(7.4)$ & $2(4.2)$ & $14(7.6)$ & \\
\hline Household income ${ }^{\ddagger}$ & & & & & $<0.001$ \\
\hline Low & $225(81.2)$ & $437(47.2)$ & $20(41.7)$ & $76(41.1)$ & \\
\hline Low-middle & 37 (13.4) & $254(27.5)$ & $12(25.0)$ & $51(27.6)$ & \\
\hline Middle-high & $9(3.2)$ & $131(14.2)$ & $10(20.8)$ & 35 (18.9) & \\
\hline High & $6(2.2)$ & $103(11.1)$ & $6(12.5)$ & $23(12.4)$ & \\
\hline Region & & & & & 0.19 \\
\hline Seoul & 31 (11.2) & $152(16.4)$ & $9(18.8)$ & 30 (16.2) & \\
\hline Metropolitan cities & $69(24.9)$ & $206(22.3)$ & $7(14.6)$ & $50(27.0)$ & \\
\hline Provinces & $177(63.9)$ & $567(61.3)$ & $32(66.7)$ & $105(57.8)$ & \\
\hline No. of chronic diseases & $1.30 \pm 1.13$ & $1.23 \pm 1.09$ & $1.60 \pm 1.09$ & $1.37 \pm 1.09$ & 0.06 \\
\hline Seasonal influenza vaccination & $219(79.1)$ & $750(81.1)$ & $34(70.8)$ & $153(82.7)$ & 0.26 \\
\hline H1N1 influenza vaccination & $164(59.2)$ & $588(63.6)$ & $21(43.8)$ & $107(57.8)$ & 0.02 \\
\hline
\end{tabular}

Values are presented as numbers (\%) or means \pm SD.

*Analysis of variance and the chi-square test were used for the statistical analysis of continuous and categorical variables, respectively. ${ }^{\dagger}$ Education levels were classified into 4 categories: completion of elementary school, middle school, high school, and post-secondary school. \#Household income levels were divided into quartiles calculated according to equalized income (total household income divided by the square root of the number of people in a household).

Table 2. Association between the type of living arrangement and non-receipt of seasonal influenza vaccination

\begin{tabular}{|c|c|c|c|c|c|c|}
\hline Living arrangement & Crude odds ratio & $95 \%$ confidence interval & P-value* & Adjusted $^{\dagger}$ odds ratio & $95 \%$ confidence interval & P-value* \\
\hline Living alone & 1 (Reference) & & & 1 (Reference) & & \\
\hline Living with a spouse & 0.88 & $0.63-1.23$ & 0.46 & 0.76 & $0.53-1.09$ & 0.14 \\
\hline Living with offspring & 1.556 & $0.78-3.09$ & 0.21 & 1.74 & $0.86-3.52$ & 0.13 \\
\hline Living with other family members & 0.79 & $0.49-1.27$ & 0.33 & 0.78 & $0.48-1.29$ & 0.33 \\
\hline
\end{tabular}

*Logistic regression analyses were used for the statistical analysis. ${ }^{\dagger}$ Adjusted for age, sex, region, education level, income level, and number of comorbidities.

Table 3. Association between the type of living arrangement and non-receipt of H1N1 influenza vaccination

\begin{tabular}{|c|c|c|c|c|c|c|}
\hline Living arrangement & Crude odds ratio & $95 \%$ confidence interval & P-value* & Adjusted $^{\dagger}$ odds ratio & $95 \%$ confidence interval & P-value* \\
\hline Living alone & 1 (Reference) & & & 1 (Reference) & & \\
\hline Living with a spouse & 0.83 & $0.63-1.10$ & 0.19 & 0.86 & $0.63-1.16$ & 0.32 \\
\hline Living with offspring & 1.87 & $1.01-3.46$ & 0.05 & 2.03 & $1.08-3.82$ & 0.03 \\
\hline Living with other family members & 1.06 & $0.73-1.54$ & 0.77 & 1.11 & $0.74-1.63$ & 0.64 \\
\hline
\end{tabular}

${ }^{*}$ Logistic regression analyses were used for the statistical analysis. ${ }^{\dagger}$ Adjusted for age, sex, region, education level, income level, and number of comorbidities.

women. Table 1 presents the sociodemographic characteristics according to the type of living arrangement. The distributions of sex, age, education, and income level differed significantly among the types of living arrangements. Those living with offspring (without spouse) had a higher number of chronic diseases. The overall seasonal influenza vaccination rate in this population was 80.6 percent, and the $\mathrm{H} 1 \mathrm{~N} 1$ influenza vaccination rate was 61.3 percent. A lower vaccination rate was observed among participants living with offspring (without spouse), whereas those living with a spouse had higher rates of both seasonal and H1N1 influenza vaccination. Table 2 and Table 3 demonstrate the association between living arrange- ments and influenza vaccination rates. For H1N1 influenza vaccination, after adjusting for age, sex, region, education level, income level, and number of comorbidities, the living with offspring (without spouse) group was less likely to have received $\mathrm{H} 1 \mathrm{~N} 1$ vaccination (odds ratio [OR], 2.03; 95\% confidence interval $[\mathrm{CI}], 1.08-3.82)$ than the living alone group. However, this association was not statistically significant for seasonal influenza vaccination (OR, 1.74; 95\% CI, 0.86-3.52).

\section{DISCUSSION}

Previous studies have investigated factors associated with influ- 
enza vaccination, such as age, sex, educational level, household income, place of residence, contact with the health care system, hospitalization, and comorbidities. We further examined the associations between the type of living arrangements and seasonal and $\mathrm{H} 1 \mathrm{~N} 1$ influenza vaccination rates among elderly South Korean individuals. This study showed that compared with the living alone group, those living with offspring (without spouse) were less likely to receive influenza vaccinations. In particular, those living with offspring (without spouse) had a statistically significantly lower rate of $\mathrm{H} 1 \mathrm{~N} 1$ influenza vaccination, even after adjusting for confounding variables. Furthermore, although these results were not statistically significant, those living with a spouse were more likely to have received influenza vaccinations than subjects in the other living arrangement groups. Our findings are similar to those of several previous studies. A previous study reported that compared with the living alone group, subjects in the living with adult offspring group were less likely to receive recommended preventive care such as influenza vaccinations and physical and dental checkups, whereas subjects in the living with a spouse only group were more likely to receive preventive care. ${ }^{15)}$ Other previous studies observed that the influenza vaccination rate among elderly subjects depended on the marital status. Whereas married subjects had the highest rate of influenza vaccination, those in the divorced or separated group had the lowest rate of influenza vaccination. ${ }^{18)}$ Similarly, another study found that married elderly subjects had a higher influenza vaccination rate, compared with the non-married elderly. ${ }^{19)}$ Similarly, several studies suggested that spouses play an important role in providing instrumental and emotional support, which affects personal health behaviors and illnesses. ${ }^{20,21)}$ However, the characteristics of a parent-offspring relationship may differ from those of a spousal relationship. Many elderly adults who live with their offspring already have health-related or economic problems. ${ }^{15)}$ Therefore, they may experience difficulty in receiving preventive care because of the burdens of costs on themselves or their offspring. Additionally, adult offspring who live with their parents might have characteristics that make them less able to provide financial and other support to their parents to ensure the receipt of appropriate preventive care.

Social network channels, which represent the web of social relationships with family members, close friends, and more formal relationships, are a good resource of health information and health practices. Elderly individuals who live with their offspring tend to have narrow social network channels that focus only on their offspring. ${ }^{22)}$ This might also explain why the results did not change when income levels were considered. Most governmental outreach interventions only target those who live alone. Because the presence of adult offspring cannot be considered a default resource from which elderly individuals can obtain preventive care, educational and outreach inter- ventions should target not only those who live alone, but also those who live with offspring.

This study has the following limitations. First, the survey asked whether participants had received the vaccination during the past year and thus could not reflect more current changes in living arrangements. Second, influenza vaccination receipt data were self-reported and thus subject to recall bias. Furthermore, a trivalent influenza vaccine that provides simultaneous protection against 3 strains at once (influenza, A/H3N2, A/H1N1, and influenza B) was first offered in $2010,{ }^{23)}$ leading to the possible failure of elderly subjects to recognize that they had received an $\mathrm{H} 1 \mathrm{~N} 1$ influenza vaccination, as the influenza and H1N1 vaccines were combined. Finally, there might have been uncontrolled residual confounding variables.

In conclusion, our findings suggest differences in the influenza vaccination rates according to the type of living arrangements. In particular, those living with offspring (without spouse) had a significantly lower H1N1 influenza vaccination rate when compared to subjects with other living arrangements. Interventions to improve influenza vaccination coverage should target not only elderly persons who live alone, but also those living with offspring.

\section{CONFLICT OF INTEREST}

No potential conflict of interest relevant to this article was reported.

\section{REFERENCES}

1. World Health Organization. Media centre: fact sheet: influenza (seasonal) [Internet]. Geneva: World Health Organization; 2014 [cited 2015 Feb 10]. Available from: http://www.who.int/mediacentre/factsheets/ fs $211 / \mathrm{en} /$.

2. Statistics Korea. The causes of death in Korea 2013 [Internet]. Daejeon: Statistics Korea [cited 2015 Feb 10]. Available from: http://kostat.go.kr.

3. Noh JY, Kim WJ. Influenza vaccines: unmet needs and recent developments. Infect Chemother 2013;45:375-86.

4. Kramarz P, Ciancio B, Nicoll A. Seasonal and pandemic influenza vaccines for the elderly and other risk groups: a review of available data. Pol Arch Med Wewn 2009;119:654-9.

5. Nichol KL, Nordin J, Mullooly J, Lask R, Fillbrandt K, Iwane M. Influenza vaccination and reduction in hospitalizations for cardiac disease and stroke among the elderly. N Engl J Med 2003;348:1322-32.

6. Hak E, Buskens E, van Essen GA, de Bakker DH, Grobbee DE, Tacken MA, et al. Clinical effectiveness of influenza vaccination in persons younger than 65 years with high-risk medical conditions: the PRISMA study. Arch Intern Med 2005;165:274-80.

7. Vaux S, Van Cauteren D, Guthmann JP, Le Strat Y, Vaillant V, de Valk H, et al. Influenza vaccination coverage against seasonal and pandemic influenza and their determinants in France: a cross-sectional survey. BMC Public Health 2011;11:30.

8. Kee SY, Lee JS, Cheong HJ, Chun BC, Song JY, Choi WS, et al. Influenza 
vaccine coverage rates and perceptions on vaccination in South Korea. J Infect 2007;55:273-81.

9. Dip RM, Cabrera MA. Influenza vaccination in non-institutionalized elderly: a population-based study in a medium-sized city in Southern Brazil. Cad Saude Publica 2010;26:1035-44.

10. Pena-Rey I, Perez-Farinos N, Sarria-Santamera A. Factors associated with influenza vaccination among elderly Spanish women. Public Health 2004;118:582-7.

11. Kang HS, Lee H, Kim MW. The correlates of influenza vaccination among Korean elderly men and women. J Korean Acad Community Health Nurs 2011;22:45-55.

12. Brien S, Kwong JC, Buckeridge DL. The determinants of 2009 pandemic A/H1N1 influenza vaccination: a systematic review. Vaccine 2012;30: 1255-64.

13. Andrew MK, McNeil S, Merry H, Rockwood K. Rates of influenza vaccination in older adults and factors associated with vaccine use: a secondary analysis of the Canadian Study of Health and Aging. BMC Public Health 2004;4:36.

14. Wiese-Posselt M, Leitmeyer K, Hamouda O, Bocter N, Zollner I, Haas $\mathrm{W}$, et al. Influenza vaccination coverage in adults belonging to defined target groups, Germany, 2003/2004. Vaccine 2006;24:2560-6.

15. Lau DT, Kirby JB. The relationship between living arrangement and preventive care use among community-dwelling elderly persons. Am J
Public Health 2009;99:1315-21.

16. Wyke S, Ford G. Competing explanations for associations between marital status and health. Soc Sci Med 1992;34:523-32.

17. Seeman TE. Social ties and health: the benefits of social integration. Ann Epidemiol 1996;6:442-51.

18. Lu PJ, Singleton JA, Rangel MC, Wortley PM, Bridges CB. Influenza vaccination trends among adults 65 years or older in the United States, 1989-2002. Arch Intern Med 2005;165:1849-56.

19. Chiatti C, Barbadoro P, Lamura G, Pennacchietti L, Di Stanislao F, D’Errico MM, et al. Influenza vaccine uptake among communitydwelling Italian elderly: results from a large cross-sectional study. BMC Public Health 2011;11:207.

20. Monden C. Partners in health?: exploring resemblance in health between partners in married and cohabiting couples. Sociol Health Illn 2007;29:391-411.

21. Liang J, Brown JW, Krause NM, Ofstedal MB, Bennett J. Health and living arrangements among older Americans: does marriage matter? J Aging Health 2005; 17:305-35.

22. Hwang MY, Lee JS. Informal support network typology of the urban low-income elderlies. Korean J Fam Soc Work 1998;2:169-93.

23. Korean Centers for Disease Control and Prevention. Notice [Internet]. Daejeon: Korean Centers for Disease Control and Prevention [cited 2015 Feb 10]. Available from: http://www.cdc.go.kr. 